\title{
Zinc-alpha2-glycoprotein in patients with acute and chronic kidney disease
}

\author{
Inga Sörensen-Zender ${ }^{\dagger}$, Jan Beneke ${ }^{\dagger}$, Bernhard MW Schmidt, Jan Menne, Hermann Haller and Roland Schmitt
}

\begin{abstract}
Background: Zinc-alpha2-glycoprotein (AZGP1) is a secreted protein which is synthesized in a variety of cell types. AZGP1 has functionally been implicated in lipid metabolism, the regulation of cell cycling and cancer progression. Previous studies have shown increased circulating AZGP1 levels in patients with chronic kidney disease but AZGP1 has not been investigated in acute kidney injury (AKI). In this study, serum AZGP1 levels were measured in acute and chronic kidney disease to test for a correlation to renal function and other clinical parameters.

Methods: We performed ELISA based measurements of AZGP1 serum levels in 21 patients suffering from grade 3 AKI and in 20 chronic hemodialysis patients. In AKI patients, AZGP1 was first measured before initiation of acute renal replacement therapy and a second measurement was done during renal functional recovery. Sera of healthy blood donors served as controls. The association of AZGP1 with acute and chronic renal dysfunction was analysed, as well as the correlation with clinical parameters, body composition and biochemical variables.

Results: Levels of circulating AZGP1 were significantly elevated in AKI patients. High initial levels of AZGP1 correlated with extra-renal complications but not with parameters of renal function. At follow-up, AZGP1 levels were still increased but now correlated significantly with creatinine, eGFR and urea. Circulating AZGP1 in chronic hemodialysis patients was higher than in AKI patients. An association to parameters of lipid metabolism was not found.

Conclusions: This study illustrates that circulating AZGP1 is not only elevated in chronic hemodialysis patients but also sharply increases during the early phase of AKI. The unexpected association with extra-renal complications during AKI needs further exploration as it might point to unknown biological effects of AZGP1.
\end{abstract}

Keywords: Zinc-alpha2-glycoprotein, Hemodialysis, Acute kidney injury, Adipokine

\section{Background}

Zinc-alpha2-glycoprotein (AZGP1) is a secreted soluble protein which is synthesized in adipocytes and a variety of other cell types [1]. Although the definite role of AZGP1 remains unclear, it has been implicated in several biological processes. Besides its potential role in regulation of cell cycling and cancer progression [1-3], AZGP1 has been characterized as a functional modifier of lipid metabolism [4,5]. In vitro, AZGP1 stimulated lipolysis in adipocytes via activation of $\alpha 3$-adrenoceptors [6]. Injections into rats triggered expression of lipolytic enzymes, resulting in increased lipid mobilization and loss of body fat [7]. In mice with cancer cachexia the

\footnotetext{
* Correspondence: schmitt.roland@mh-hannover.de

${ }^{\dagger}$ Equal contributors

Department of Nephrology, Hannover Medical School, Carl-Neuberg-Str. 1, Hannover D-30625, Germany
}

expression of AZGP1 was up-regulated suggesting that AZGP1 might act as a cancer cachexia factor [5]. Genetic deletion of AZGP1 resulted in increased body weight and reduced lipolytic capacity [8].

In 2008 an ELISA was developed which allowed measurement of AZGP1 levels in patient serum [9]. Several studies have since been conducted determining serum AZGP1 in different patient populations. While Stejskal et al. did not find significantly altered levels of AZGP1 in patients with metabolic syndrome [9], Yeung et al. showed a positive correlation between serum AZGP1 and BMI, insulin resistance and serum triglycerides [10]. Other conditions associated with increased serum AZGP1 levels included preeclampsia [11] and chronic heart failure [12], whereas decreased levels of AZGP1 were found in HIV patients [13]. 
Although some of these studies argued for an involvement of AZGP1 in changes of lipid metabolism, a clear causeeffect relationship has not been shown. Dysregulated lipid metabolism is thought to contribute importantly to the development of insulin resistance and increased mortality in patients with chronic kidney disease (CKD) [14]. Two recent studies explored serum AZGP1 levels in chronic hemodialysis (HD) patients and found significantly increased mean values $[15,16]$. Philipp et al. suggested that this increase was most likely caused by a loss of normal renal clearance of the protein [16]. While they could not find a correlation between AZGP1 levels and parameters of lipid metabolism or body composition [16], the second study showed an inverse correlation between circulating AZGP1 and adiposity [17]. So far, AZGP1 has not been investigated in the context of acute kidney injury (AKI).

In the present study we measured circulating AZGP1 in patients with $\mathrm{AKI}$ and in chronic $\mathrm{HD}$ patients to further explore AZGP1 in renal dysfunction.

\section{Methods \\ Patients}

We studied 20 adult patients on maintenance HD and 21 adult AKI patients admitted to Hannover Medical School during the EHEC serotype O104:H4 outbreak in 2011 [18]. Preexisting disease was systematically recorded and patients were classified into having comorbidities if diabetes, hypertension or coronary heart disease were present. Only patients with documented normal baseline renal function were included. All evaluated patients suffered from grade 3 AKI, according to the Acute Kidney Injury Network (AKIN) classification and required transient renal replacement therapy. All patients consented in writing to donating blood for scientific evaluation. Serum from 20 healthy subjects served as control and was obtained from blood donors at the Institute of Transfusion Medicine, Hannover Medical School. The protocol was carried out according to the Declaration of Helsinki and approved by the Institutional Review Board at Hannover Medical School. Standard laboratory values were measured at the central clinical laboratory.

\section{Quantification of circulating AZGP1 and other biochemical variables}

Blood samples were obtained after an overnight-fast before $\mathrm{HD}$ in chronic $\mathrm{HD}$ patients. In AKI patients, blood was drawn before initiation of the first HD session as baseline value and follow-up samples were gathered 85 days in average after the last dialysis session. Serum AZGP1 was measured by a commercial enzyme-linked immunosorbent assay (Biovendor, Modrice, Czech Republic), according to the manufacturer's instructions by investigators blinded to patients' data. All measurements were performed in duplicate. The assay sensitivity was $0.673 \mathrm{ng} / \mathrm{ml}$. The intraassay coefficient of variation was less than 5\%. Serum creatinine, urea, cholesterol, triglycerides, CRP, leukocytes, AST, ALT and LDH were measured by standard laboratory methods in a certified laboratory.

\section{Clinical data collection}

Age, gender and BMI were recorded in all patients and additional data were retrieved from patient charts. BMI was calculated with pre-dialysis body weight. Total body fat in maintenance HD patients was measured using standard multi-frequency bioelectrical impedance analysis at 30 minutes after a midweek dialysis session as previously described [19]. For AKI patients, data were retrieved from the centralized STEC-HUS consortium database which has previously been described [18]. eGFR was calculated using the CKD-EPI equation [20].

\section{Statistical analysis}

Continuous variables are presented as median (interquartile range). Nonparametric ANOVA (Kruskall-Wallis test) was performed if more than 2 groups were analyzed. In this case Mann-Whitney $U$ test was performed as posthoc test with a p-value $<0.025$ as significance level. Otherwise data were compared using Mann-Whitney U or Wilcoxon test employing Spearman tests as appropriate. P-values $<0.05$ were considered significant. Stepwise linear regression analysis with forward selection was performed to identify parameters independently predicting AZGP levels at follow-up.

\section{Results}

All AKI patients included in our study were hospitalized for Shiga-toxin induced haemolytic uraemic syndrome (STEC-HUS) during the german outbreak 2011. AZGP1 was significantly higher in AKI patients than in control subjects (Figure 1A). In control subjects we found no correlation of AZGP1 to creatinine (82.3 (66.95 - 94.8) $\mu \mathrm{mol} / \mathrm{l})$ or eGFR $(77.8(66.9$ - 91.3) $\mathrm{ml} / \mathrm{min})$. Similarly, among AKI patients, we found no significant correlation of initial AZGP1 to creatinine, urea and eGFR (Table 1). Initial AZGP1 levels higher or equal to median $(121.87 \mu \mathrm{g} / \mathrm{ml})$ positively correlated with age, pre-existing comorbidities and a more severe outcome of STEC-HUS (Table 2). All patients requiring ventilation belonged to this subgroup and patients of this group developed more often neurological symptoms including seizures and pathological EEGs (Table 2, Figure 1B).

Kidney function in all AKI patients recovered until they were discharged after $34(27$ - 38) hospital days, as judged by lack of need for further renal replacement therapy. AZGP1 levels were determined again in a follow-up ambulatory visit at $85(44-100)$ days after the initial 

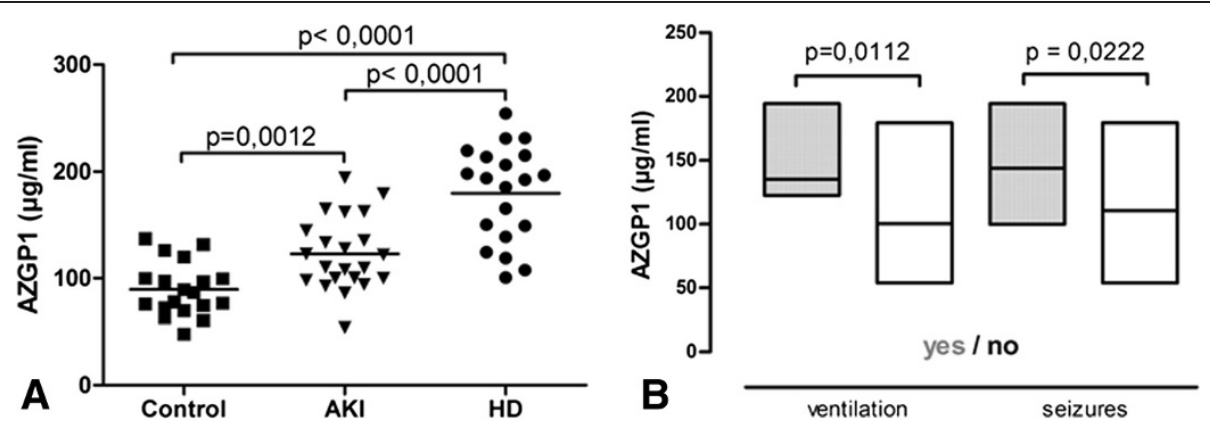

Figure 1 AZGP1 serum levels are elevated in acute and chronic renal failure and correlate with extra-renal complications during AKI. A: Mean serum AZGP1 serum levels were significantly higher in the AKI cohort than in controls at the time before the first session of dialysis but lower than in chronic HD patients. B: AZGP1 levels at admission significantly differed in patients who later would or would not require ventilation or developed seizures due to STEC-HUS induced cerebral changes.

measurement (Figure 2A). At this time $50 \%$ of AKI patients showed a further increase in individual AZGP1 levels while $25 \%$ had unchanged levels and $25 \%$ a decrease in circulating AZGP1. Mean AZGP1 values did not significantly change but we found an inverse correlation between the time after initial measurement and individual follow-up levels $(\mathrm{r}=-0.6125 ; \mathrm{p}=0.0032)$. AZGP1 at follow-up correlated with increased creatinine, urea and eGFR (Figure 2B - C, Table 1). Higher AZGP1 was also associated with increased AST, ALT and LDH (Table 1).

Stepwise linear regression analysis with forward selection revealed serum creatinine $(\beta=.605, p=0.005)$ and LDH $(\beta=.185, \mathrm{p}=0.022)$ as only factors independently associated with AZGP1. Patients presenting serum creatinine levels within normal limits $(<84 \mu \mathrm{mol} / \mathrm{l}$ for females, $<104 \mu \mathrm{mol} / \mathrm{l}$ for males) showed significantly lower AZGP1 values (113.69 (104.65 - 128.42) $\mu \mathrm{g} / \mathrm{ml})$ as compared to patients with incomplete renal recovery (158.17 (127.44 - 176.69) $\mu \mathrm{g} / \mathrm{ml} ; \mathrm{p}=0.012)$.

End stage renal disease has been shown to be associated with increased AZGP1 levels [15,16]. We confirmed this finding in a cohort of 20 chronic HD patients (Figure 1A). In these patients circulating AZGP1 levels where higher than in the AKI cohort. Given the suggested role of AZGP1 in lipid metabolism and body composition we analyzed AZGP1 levels with regard to age, BMI, total body fat as measured by bioelectrical impedance analysis, triglycerides, LDL, HDL, total cholesterol and total protein. In our cohort AZGP1 levels did not associate with any of these parameters (Table 3).

\section{Discussion}

Our study is the first to assess AZGP1 levels in AKI patients. We studied patients of the STEC-HUS cohort who presented with a very rapid and homogenous onset of AKI [18]. We found that the acute rise of serum creatinine in these patients was paralleled by a sharp increase in circulating AZGP1. Patients with stronger elevations of AZGP1 were older and developed significantly more extra-renal complications such as seizures or the requirement of mechanical ventilation. While follow-up AZGP1 levels correlated inversely with parameters of renal recovery they correlated positively with serum markers of cell damage.

An interesting finding of our study was that higher initial levels of AZGP1 correlated with a more severe outcome and with extra-renal complications in STEC-HUS

Table 1 Univariate correlation between serum AZGP1 and other variables

\begin{tabular}{|c|c|c|c|c|}
\hline & \multicolumn{2}{|r|}{ AKI } & \multicolumn{2}{|c|}{ AKI follow-up } \\
\hline & \multicolumn{2}{|r|}{$\mathrm{n}=21$} & \multicolumn{2}{|r|}{$n=21$} \\
\hline & Median (quantiles) & Spearmans' coefficient; p-value & Median (quantiles) & Spearmans' coefficient; p-value \\
\hline creatinine $(\mu \mathrm{mol} / \mathrm{l})$ & $290(218-409)$ & $r=0.093 ; p=0.696$ & $101(79-132)$ & $r=0.776 ; p=<0.001$ \\
\hline eGFR (ml/min) & $16(10.5-30)$ & $r=-0.176 ; p=0.458$ & $52(44-81)$ & $r=-0.777 ; p=<0.001$ \\
\hline urea $(\mathrm{mmol} / \mathrm{l})$ & $15.8(1.9-28.5)$ & $r=0.144 ; p=0.546$ & $7.6(5.3-9.9)$ & $r=0.659 ; p=0.002$ \\
\hline LDH $(\mathrm{U} / \mathrm{I})$ & $1311(871-1791)$ & $r=-0.168 ; p=0.478$ & $192(171-237)$ & $r=0.887 ; p=<0.0001$ \\
\hline CRP $(\mathrm{mg} / \mathrm{l})$ & $40.3(21.8-101.8)$ & $r=-0.104 ; p=0.663$ & $1(1-6.5)$ & $r=0.62 ; p=0.048$ \\
\hline AST $(U / I)$ & $68(58-89)$ & $r=-0.139 ; p=0.621$ & $23(20-26.5)$ & $r=0.49 ; p=0.064$ \\
\hline $\operatorname{ALT}(\mathrm{U} / \mathrm{I})$ & $32.5(23-47.5)$ & $r=-0.075 ; p=0.775$ & $17(12-23.5)$ & $r=0.67 ; p=0.006$ \\
\hline leukocytes $\left(10^{3} / \mu \mathrm{l}\right)$ & $14.8(8.8-19.6)$ & $r=0.390 ; p=0.089$ & $5.6(4.8-6.7)$ & $r=0.455 ; p=0.044$ \\
\hline
\end{tabular}


Table 2 Difference between AKI patients with AZGP1 higher/lower than median

\begin{tabular}{|c|c|c|c|}
\hline Admission AZGP1 & $\begin{array}{c}\geq \text { median } \\
n=11\end{array}$ & $\begin{array}{c}<\text { median } \\
n=10\end{array}$ & p-value \\
\hline \multicolumn{4}{|l|}{ basic parameters } \\
\hline age (years) & $54(48-70)$ & $37(23-46)$ & 0.039 \\
\hline $\begin{array}{l}\text { comorbidities, n (\%) hypertension, } \\
\text { diabetes mellitus, coronary } \\
\text { heart disease }\end{array}$ & $7(63.6)$ & $1(10)$ & 0.01 \\
\hline \multicolumn{4}{|l|}{ outcome parameters } \\
\hline ventilation, $\mathrm{n}(\%)$ & $7(63.6)$ & $0(0)$ & $<0.001$ \\
\hline seizures, n (\%) & $7(63.6)$ & $1(10)$ & 0.01 \\
\hline EEG pathological, n (\%) & $10(90.9)$ & $4(40)$ & 0.012 \\
\hline
\end{tabular}

AKI. This increase in circulating AZGP1 might either result from enhanced synthesis and cellular release or it could reflect disrupted elimination. While there is no indication that AZGP1 synthesis is increased during acute illness there is data from almost 40 years ago suggesting that renal function and serum AZGP1 levels are inversely related [21]. Along these lines, it has been noted by Philipp et al. that enhanced AZGP1 levels in CKD patients might be best explained by an accumulation effect due to the loss of normal renal elimination [16]. It has also been suggested that increased levels of AZGP1 in heart failure patients might be due to diminished renal function [12].

With a size of $40 \mathrm{kD}$ AZGP1 is predicted to be normally filtered in the glomerulum and thereafter cleared by the proximal tubule through reabsorption and lysosomal degradation [22]. Accordingly, our results show that the acute elevation in AZGP1 during AKI persisted if renal recovery was incomplete. Highest levels were observed in chronic HD patients pointing to a maximal accumulative effect. In the AKI cohort we observed an association between initial AZGP1 levels and pre-existing comorbidities, such as diabetes, hypertension and coronary heart disease. Although all AKI patients had normal documented prior creatinine values our study can not exclude subclinical renal impairment. The general lack of correlation between renal functional parameters and AZGP1 during early AKI might reflect the fact that this phase of AKI is characterized by rapid dynamic changes and significantly altered kinetics for creatinine and urea that are highly variable until a steady-state is reached.

Adipose tissue is in crosstalk with many organ systems through the secretion of adipokines. A growing number of adipokines, such as leptin and adiponectin, has been identified and a mechanistic link to the pathogenesis of insulin resistance, metabolic syndrome and dyslipidaemia has been established [23,24]. AZGP1 which is also expressed
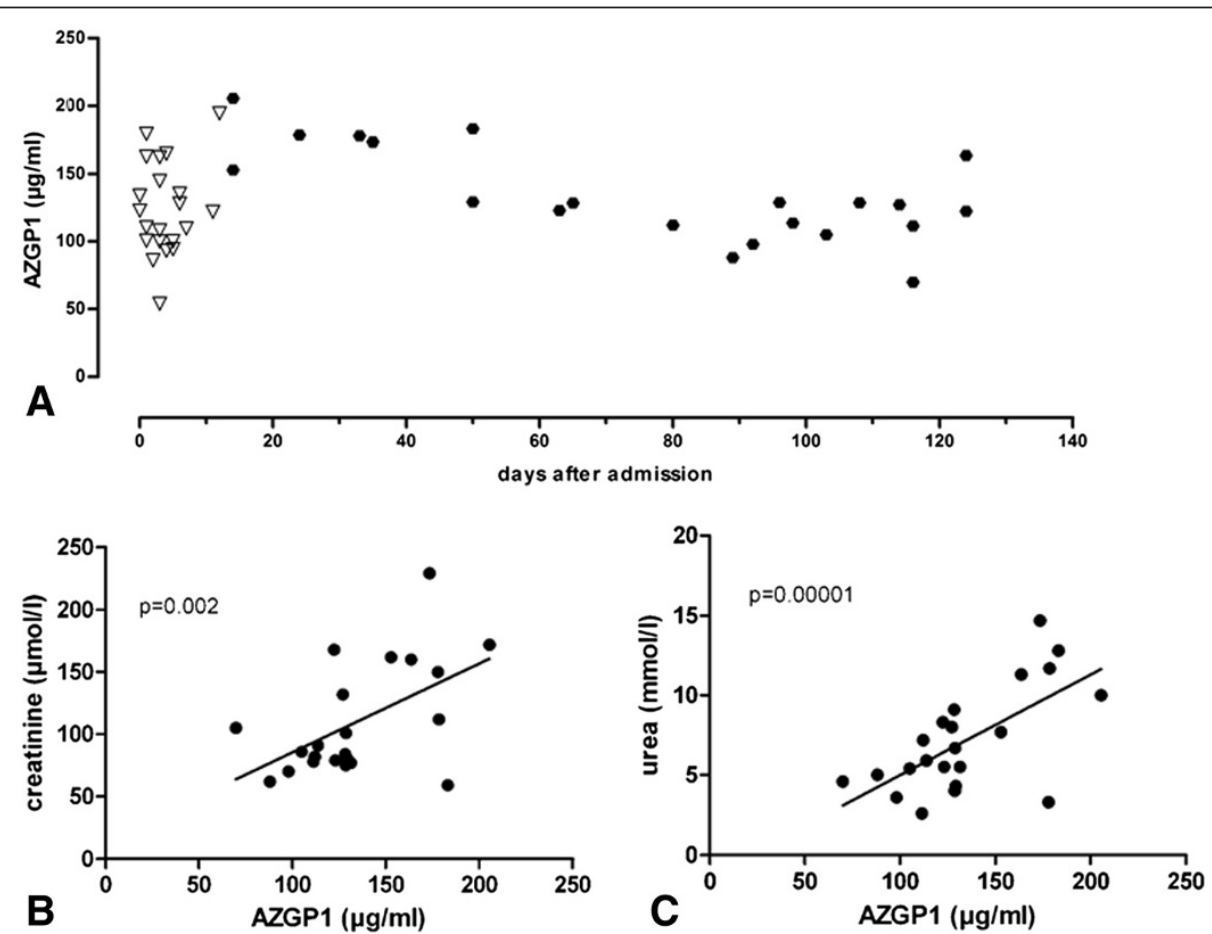

Figure 2 AZGP1 serum levels correlate with parameters of renal function at follow-up measurements after AKI. A: AZGP1 serum level s in patients by days after admission to hospital. Initial measurements are depicted as triangles, follow-up measurements as circles. B,C: Mean AZGP1 levels at follow-up (85 (44 - 100) days after the initial measurement) significantly correlated with creatinine and urea. 
Table 3 Univariate correlation between serum AZGP1 levels and other variables in chronic HD patients

\begin{tabular}{lcc}
\hline & \multicolumn{2}{c}{ Chronic HD patients } \\
\cline { 2 - 3 } & \multicolumn{2}{c}{$\mathbf{n = 2 0}$} \\
\cline { 2 - 3 } & $\begin{array}{c}\text { Median (quantiles) } \\
\text { age (years) }\end{array}$ & $\begin{array}{c}\text { Pearsons's coefficient; } \\
\text { p-value }\end{array}$ \\
\hline BMI & $25.9 \pm 5$ & $r=-0.2 ; p=0.323$ \\
total body fat (\%) & $34 \pm 9$ & $r=-0.28 ; p=0.23$ \\
triglycerides (mg/dl) & $194(122-302)$ & $r=-0.07 ; p=0.721$ \\
LDL (mg/dl) & $101(80-109)$ & $r=-0.003 ; p=0.81$ \\
HDL $(\mathrm{mg} / \mathrm{dl})$ & $41(30-48)$ & $r=0.05 ; p=0.81$ \\
total cholesterol & $178(148-218)$ & $r=0.133 ; p=0.587$ \\
(mg/dl) & & $r=-0.204 ; p=0.307$ \\
total protein $(\mathrm{g} / \mathrm{l})$ & $6.8(6.6-7)$ &
\end{tabular}

in adipocytes was suggested to act as a novel adipokine [25]. In human visceral and subcutaneous fat AZGP1 expression is negatively associated with increased adiposity and the parameters of insulin resistance [25]. These observations lead to the hypothesis that AZGP1 might be protective in obese individuals exerting anti-obese and anti-diabetic effects [26]. In agreement with two previous reports [15,16], we observed substantially higher levels of AZGP1 in CKD patients. However, we found no correlation between individual AZGP1 levels and body fat, BMI, triglycerides or cholesterol. We can not rule out that a broader analysis including lipid metabolites such as oxidized LDL might have revealed AZGP1 associated changes. An additional limitation of our study is the small sample size which reduced the power to detect more subtle associations.

\section{Conclusion}

In summary, acute and chronic loss of renal function is associated with a significant increase in circulating AZGP1. The most likely reason is an imbalance between endogenous production and physiological elimination of the protein by the kidney. In the STEC-HUS AKI cohort initial AZGP1 levels correlated with the severity of the disease course and extra-renal complications. This might be explained by pre-existing subclinical renal functional impairment in patients who were sicker but could also imply additional pathophysiological effects of AZGP1. In chronic HD patients we found no association of individual AZGP1 levels to parameters of fat metabolism. Further research is needed to explore additional potential effects of increased AZGP1 in patients with kidney disease.

\section{Competing interests}

The authors have no competing interests.

\section{Authors' contributions}

IS-Z and RS designed the study. IS-Z, JM and JB collected blood samples and performed the AZGP1 measurements. HH, JM, IS-Z, JB, BMWS and RS evaluated the data and wrote the manuscript. All authors read and approved the final manuscript.

\section{Acknowledgements}

This study was supported by a grant from the Deutsche

Forschungsgemeinschaft (SCHM 2146/3-1).

Received: 6 May 2013 Accepted: 1 July 2013

Published: 12 July 2013

\section{References}

1. Hassan MI, Waheed A, Yadav S, Singh TP, Ahmad F: Zinc alpha 2-glycoprotein: a multidisciplinary protein. Molecular cancer research: MCR 2008, 6(6):892-906.

2. Lei G, Brysk H, Arany I, Tyring SK, Srinivasan G, Brysk MM: Characterization of zinc-alpha(2)-glycoprotein as a cell adhesion molecule that inhibits the proliferation of an oral tumor cell line. J Cell Biochem 1999, 75(1):160-169.

3. Schmitt R, Marlier A, Cantley LG: Zag expression during aging suppresses proliferation after kidney injury. Journal of the American Society of Nephrology: JASN 2008, 19(12):2375-2383.

4. Bao $Y$, Bing $C$, Hunter $L$, Jenkins JR, Wabitsch $M$, Trayhurn P: Zinc-alpha2-glycoprotein, a lipid mobilizing factor, is expressed and secreted by human (SGBS) adipocytes. FEBS Lett 2005, 579(1):41-47.

5. Bing C, Bao Y, Jenkins J, Sanders P, Manieri M, Cinti S, Tisdale MJ, Trayhurn P: Zinc-alpha2-glycoprotein, a lipid mobilizing factor, is expressed in adipocytes and is up-regulated in mice with cancer cachexia. Proc Natl Acad Sci U S A 2004, 101(8):2500-2505.

6. Russell ST, Zimmerman TP, Domin BA, Tisdale MJ: Induction of lipolysis in vitro and loss of body fat in vivo by zinc-alpha2-glycoprotein. Biochim Biophys Acta 2004, 1636(1):59-68.

7. Russell ST, Tisdale MJ: Studies on the anti-obesity activity of zinc-alpha2-glycoprotein in the rat. Int J Obes (Lond) 2011, 35(5):658-665.

8. Rolli V, Radosavljevic M, Astier V, Macquin C, Castan-Laurell I, Visentin V, Guigne C, Carpene C, Valet P, Gilfillan S, et al: Lipolysis is altered in MHC class I zinc-alpha(2)-glycoprotein deficient mice. FEBS Lett 2007, 581(3):394-400.

9. Stejskal D, Karpisek M, Reutova H, Stejskal P, Kotolova H, Kollar P: Determination of serum zinc-alpha-2-glycoprotein in patients with metabolic syndrome by a new ELISA. Clin Biochem 2008, 41(4-5):313-316

10. Yeung DC, Lam KS, Wang Y, Tso AW, Xu A: Serum zinc-alpha2-glycoprotein correlates with adiposity, triglycerides, and the key components of the metabolic syndrome in Chinese subjects. J Clin Endocrinol Metab 2009, 94(7):2531-2536.

11. Stepan H, Philipp A, Roth I, Kralisch S, Jank A, Schaarschmidt W, Lossner U, Kratzsch J, Bluher M, Stumvoll M, et al: Serum levels of the adipokine zinc-alpha2-glycoprotein are increased in preeclampsia. J Endocrinol Invest 2012, 35(6):562-565.

12. Tedeschi S, Pilotti E, Parenti E, Vicini V, Coghi P, Montanari A, Regolisti G, Fiaccadori E, Cabassi A: Serum adipokine zinc alpha2-glycoprotein and lipolysis in cachectic and noncachectic heart failure patients: relationship with neurohormonal and inflammatory biomarkers. Metab Clin Exp 2012, 61(1):37-42

13. Ceperuelo-Mallafre V, Escote $X$, Vilades C, Peraire J, Domingo P, Solano E, Sirvent JJ, Pastor R, Tinahones F, Leal M, et al: Zinc alpha-2 glycoprotein is implicated in dyslipidaemia in HIV-1-infected patients treated with antiretroviral drugs. HIV Med 2012, 13(5):297-303.

14. Slee AD: Exploring metabolic dysfunction in chronic kidney disease. Nutr Metab 2012, 9(1):36.

15. Leal VO, Lobo JC, Stockler-Pinto MB, Farage NE, Abdalla DS, Junior ML Mafra D: Is zinc-alpha2-glycoprotein a cardiovascular protective factor for patients undergoing hemodialysis? Clinica chimica acta; international journal of clinical chemistry 2012, 413(5-6):616-619. 
16. Philipp A, Kralisch S, Bachmann A, Lossner U, Kratzsch J, Bluher M, Stumvoll M, Fasshauer M: Serum levels of the adipokine zinc-alpha2-glycoprotein are increased in chronic hemodialysis. Metab Clin Exp 2011, 60(5):669-672.

17. Leal VO, Lobo JC, Stockler-Pinto MB, Farage NE, Velarde GC, Fouque D, Leite M Jr, Mafra D: Zinc-alpha2-Glycoprotein: Is There Association between This New Adipokine and Body Composition in Hemodialysis Patients? Ren Fail 2012, 34(9):1062-1067.

18. Menne J, Nitschke M, Stingele R, Abu-Tair M, Beneke J, Bramstedt J, Bremer JP, Brunkhorst R, Busch V, Dengler R, et al: Validation of treatment strategies for enterohaemorrhagic Escherichia coli 0104:H4 induced haemolytic uraemic syndrome: case-control study. BMJ 2012, 345:e4565.

19. David S, Kumpers P, Eisenbach GM, Haller H, Kielstein JT: Prospective evaluation of an in-centre conversion from conventional haemodialysis to an intensified nocturnal strategy. Nephrology, dialysis, transplantation: official publication of the European Dialysis and Transplant Association - European Renal Association 2009, 24(7):2232-2240.

20. Levey AS, Stevens LA: Estimating GFR using the CKD Epidemiology Collaboration (CKD-EPI) creatinine equation: more accurate GFR estimates, lower CKD prevalence estimates, and better risk predictions. American journal of kidney diseases: the official journal of the National Kidney Foundation 2010, 55(4):622-627.

21. Ekman $R$, Johansson $B G$, Ravnskov U: Renal handling of $\mathrm{Zn}$-alpha2-glycoprotein as compared with that of albumin and the retinol-binding protein. J Clin Invest 1976, 57(4):945-954.

22. Tojo A, Kinugasa S: Mechanisms of glomerular albumin filtration and tubular reabsorption. International journal of nephrology 2012, 2012:481520.

23. Kadowaki T, Yamauchi T, Kubota N, Hara K, Ueki K, Tobe K: Adiponectin and adiponectin receptors in insulin resistance, diabetes, and the metabolic syndrome. J Clin Invest 2006, 116(7):1784-1792.

24. Guzik TJ, Mangalat D, Korbut R: Adipocytokines - novel link between inflammation and vascular function? Journal of physiology and pharmacology: an official journal of the Polish Physiological Society 2006, 57(4):505-528

25. Mracek T, Stephens NA, Gao D, Bao Y, Ross JA, Ryden M, Arner P, Trayhurn P, Fearon KC, Bing C: Enhanced ZAG production by subcutaneous adipose tissue is linked to weight loss in gastrointestinal cancer patients. Br J Cancer 2011, 104(3):441-447.

26. Russell ST, Tisdale MJ: Role of beta-adrenergic receptors in the anti-obesity and anti-diabetic effects of zinc-alpha2-glycoprotien (ZAG). Biochim Biophys Acta 2012, 1821(4):590-599.

\section{doi:10.1186/1471-2369-14-145}

Cite this article as: Sörensen-Zender et al:: Zinc-alpha2-glycoprotein in patients with acute and chronic kidney disease. BMC Nephrology 2013 14:145.

\section{Submit your next manuscript to BioMed Central and take full advantage of:}

- Convenient online submission

- Thorough peer review

- No space constraints or color figure charges

- Immediate publication on acceptance

- Inclusion in PubMed, CAS, Scopus and Google Scholar

- Research which is freely available for redistribution 